\title{
Research and Application of Hybrid Adaptive Forecasting Method Based on SOA
}

\author{
Ding $\operatorname{Liu}^{1, *}$ \\ ${ }^{1}$ Hunan University of Information Technology School of Electronic Information
}

\begin{abstract}
The current network security situation is becoming more and more severe. In order to improve the accuracy of network security situation prediction, a network security situation prediction method based on crowd search algorithm optimized BP neural network is proposed. This algorithm uses the four characteristics of egoism, altruism, pre-action and uncertain reasoning unique to the crowd search algorithm to determine the search strategy, finds the best fitness individual, obtains the optimal weights and thresholds, and then performs random initialization of the BP neural network The threshold and weight are assigned, and the predicted value is obtained after neural network training. Finally, it is compared with the predicted value obtained by the other two optimization algorithms. Experiments show that the algorithm used in network security situation prediction has higher accuracy, smaller errors, and better stability.
\end{abstract}

\section{Introduction}

In recent years, a lot of research has been done in the network security situation prediction in China, and a large number of network security situation prediction models and methods have been proposed[1-3]. Many researchers try to introduce artificial intelligence methods into the field of situational awareness to improve the accuracy of situation assessment and prediction. Compared with traditional methods, the improved methods have improved the effect of evaluation and prediction to a certain extent, but these methods face

The massive data collected is not ideal in terms of accuracy and efficiency, and cannot adapt to the dynamic and changing network security requirements[46].Aiming at the problem of low accuracy of current security situation prediction, this paper combines the crowd search algorithm (SOA) with the BP neural network based on the existing network security situation prediction methods and models[7-8]. Network security posture test methods for research and simulation experiments[9].

\section{HYBRID ADAPTIVE SECURITY SITUATION PREDICTION BASED ON SOA ALGORITHM TO OPTIMIZE BP NEURAL NETWORK}

The main steps of the SOA algorithm to optimize the security situation prediction of the BP neural network (BP neural network) are as follows:

(1) On the basis of the initial population size, calculate the maximum number of iterations and spatial dimensions, and calculate the fitness of each individual.

(2) Find the individual with the best fitness. Find the global best, individual best, individual best fitness value, and global best fitness value. The most important thing in this step is the design of the fitness function, which is obtained from the BP neural network training set in this experiment.The error is taken as the fitness value.

(3) Iterative optimization. First initialize the empirical gradient direction, search step length and direction, and the parameters of the Gaussian function. Then enter the iterative loop. The termination condition of the loop is the maximum number of iterations and the double-layer loop of the population size. Determine the search direction, which can be obtained according to calculation; determine the direction of the empirical gradient, which can be obtained according to calculation; determine the parameters of the Gaussian function, which can be obtained according to calculation; determine the size of the search step, which can be obtained according to calculation; according to the calculated step sum The direction updates the position according to the formula, updates the individual optimal and best fitness values, and also updates the global optimal and fitness values. Iterate the loop until the termination condition is met, and output the global best fitness value.

\footnotetext{
* Corresponding author: ding liu@163.com
} 
(4) Obtain the best network weight and threshold. Assign the optimal weights and thresholds to random initial values for network security training.

(5) BP neural network training. Initialize the network parameters, create a neural network, call the algorithm to train the BP network, use the BP neural network for simulation prediction, calculate errors, and analyze the results. The SOA algorithm optimizes the security situation prediction algorithm flow of BP neural network as shown in Figure 1.

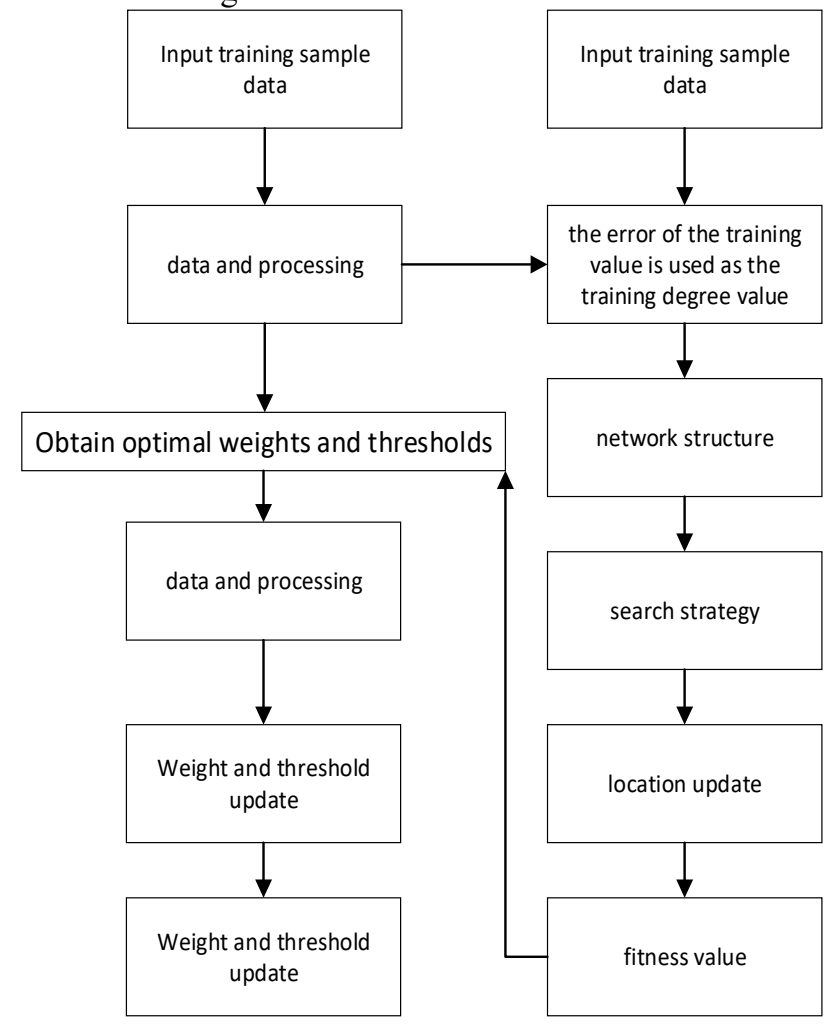

Figure 1 forecast flow chart

\section{Predictive simulation and analysis}

Figures and tables, as originals of good quality and well contrasted, are to be in their final form, ready for reproduction, pasted in the appropriate place in the text. Try to ensure that the size of the text in your figures is approximately the same size as the main text (10 point). Try to ensure that lines are no thinner than 0.25 point.

The SOA algorithm optimization method of BP neural network is applied to network security situation prediction to improve the accuracy of prediction. The experiment uses the data released in the network security information and dynamic weekly report as the experimental data. It mainly includes the number of hosts infected with the virus, the total number of websites that have been tampered with, the total number of implanted backdoor websites, the number of counterfeit pages of domestic websites, and the new information security The number of vulnerabilities is an evaluation index, which can more comprehensively reflect the status of modern network security, and can be used as an index to evaluate the basic situation of network security every week. In this experiment, the network security data information from the first period of 2015 to the seventh period of 2017 is selected. For the convenience of the experiment, we converted the five security levels into digital levels, as shown in Table 1.

Table 1 Network security situation value conversion table

\begin{tabular}{|l|l|l|l|l|}
\hline excellent & good & intermediate & poor & danger \\
\hline 5 & 4 & 3 & 2 & 1 \\
\hline
\end{tabular}

(1) data preprocessing

The data in the real world is generally incomplete, inconsistent and dirty data, which cannot be directly mined, or the mining results are not satisfactory. In order to improve the quality of data mining, data preprocessing technology is produced. There are many methods for data preprocessing: data cleaning, data integration, data transformation, data reduction, etc. These data processing techniques are used before data mining, which greatly improves the quality of data mining models and reduces the time required for actual mining. The original data should be reviewed mainly from two aspects: completeness and accuracy. The completeness audit is mainly to check whether the unit or individual that should be investigated has omissions, and whether all the survey items or indicators are filled in completely. Accuracy review mainly includes two aspects: one is to check whether the data truly reflects the objective reality and whether the content is in line with reality; the other is to check whether the data is wrong and whether the calculation is correct. The methods of reviewing data accuracy mainly include logic checking and calculation checking. Logical inspection is mainly to check whether the data conforms to logic, whether the content is reasonable, and whether there is any contradiction between items or numbers. This method is mainly suitable for the audit of qualitative (quality) data. Calculation check is to check whether the data in the questionnaire has any errors in the calculation results and calculation methods. It is mainly used for the review of quantitative (numerical) data.

For second-hand information obtained through other channels, in addition to reviewing its completeness and accuracy, the applicability and timeliness of the data should also be reviewed. Second-hand information can come from multiple sources. Some data may be obtained through special investigations for specific purposes, or may have been processed for specific purposes. For users, they should first clarify the source of the data, the caliber of the data, and the relevant background information in order to determine whether the data meets the needs of their own analysis and research, whether they need to be reprocessed, etc., and they should not be blindly copied. In addition, the timeliness of the data must be reviewed. For some issues with strong timeliness, if the data obtained is too late, it may lose the significance of the research. In general, the latest statistics should be used whenever possible. After the data has been reviewed and confirmed to be suitable for actual needs, further processing is necessary.

(2) data analysis

The purpose of data analysis is to concentrate and refine the information hidden in a large number of 
seemingly chaotic data, so as to find out the inner law of the research object. In practical applications, data analysis can help people make judgments in order to take appropriate actions. Data analysis is the process of organizing and purposefully collecting data, analyzing data, and turning it into information. This process is the supporting process of the quality management system. In the entire life cycle of the product, including every process from market research to after-sales service and final disposal, data analysis processes need to be appropriately used to improve effectiveness. For example, before starting a new design, designers must conduct extensive design investigations and analyze the data obtained to determine the design direction. Therefore, data analysis has an extremely important position in industrial design.

Online data analysis is also called online analysis and processing, which is used to process users' online requests. It has relatively high requirements for response time (usually no more than a few seconds). Compared with offline data analysis, online data analysis can process user requests in real time, allowing users to change the constraints and restrictions of analysis at any time. Compared with offline data analysis, the amount of data that can be processed by online data analysis is much smaller, but with the development of technology, the current online analysis system has been able to process tens of millions or even hundreds of millions of records in real time. The traditional online data analysis system is built on a data warehouse with a relational database as the core, while the online big data analysis system is built on the NoSQL system of the cloud computing platform. If there is no online analysis and processing of big data, there will be no way to store and index a huge number of Internet webpages, there will not be today's efficient search engines, nor will there be microblogs, blogs, and social networks built on the basis of big data processing And so on.

(3) data conversion

Attention must be paid to data conversion, and conversion cannot be carried out desperately, because sometimes conversion will seriously distort the connotation of the data itself. Example: Box-Cox conversion must be used with caution. This method that can convert almost all data will change the original form of the data. For example, the average of the first group is greater than the second group, but after conversion, the second group of data can be used No difference. Even get the opposite result. Therefore, overly complicated conversion methods cannot be used. However, in many cases, proper conversion is a good way.

(4) forecast result analysis

Aiming at the problem that the accuracy of current network security situation prediction is not high, on the basis of using SOA to optimize the weights and thresholds of BP neural network, a network security situation prediction method based on SOABP neural network is proposed. Contrast with PSO_BP optimization algorithm and GA_BP optimization algorithm. Experiments show that $\overline{\text { this }}$ algorithm can more accurately predict the future network security situation, as shown in table 2 .

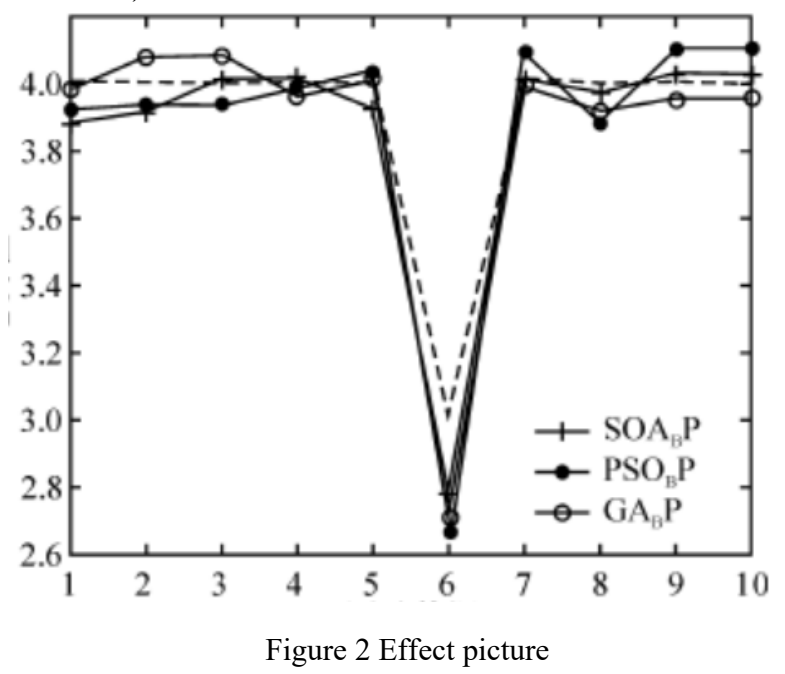

\section{In Conclusion}

The error between the predicted value and the true value of the SOA optimized BP neural network hybrid method is smaller than that of the PSO optimization and GA optimized BP neural network, and the first few predicted values obtained by the SOA algorithm optimization have a larger error with the true value, but the latter Several gradually stabilized, approaching the true value, and the curve fluctuations were relatively small. This shows that the SOA optimized BP neural network algorithm is more stable and more accurate than the other two algorithms.

\section{References}

[1]. Atmosphere Research; Research on Atmosphere Research Published by Researchers at University of Castilla-La Mancha (Evaluation of the SOA Formation in the Reaction of Furfural with Atmospheric Oxidants)[J]. Science Letter,2020.

[2]. Urban Research - Urban Planning; Reports Outline Urban Planning Findings from SOA University (An Empirical Model for Indian Senior Citizens In Traffic Management)[J]. Politics \&amp; Government Week,2020.

[3]. Physics; Universiti Tenaga Nasional Researchers Add New Study Findings to Research in Physics (Tunable multiwavelength fiber laser based on bidirectional SOA in conjunction with Sagnac loop mirror interferometer)[J]. Physics Week,2020.

[4]Olli-Pekka Tikkanen,Angela Buchholz,Arttu Ylisirniö,Siegfried Schobesberger,Annele Virtanen,Taina Yli-Juuti. Comparing secondary organic aerosol (SOA) volatility distributions derived from isothermal SOA particle evaporation data and FIGAERO-CIMS measurements[J]. Atmospheric Chemistry and Physics,2020,20(17).

[5]Iida Pullinen,Sebastian Schmitt,Sungah Kang,Mehrnaz Sarrafzadeh,Patrick Schlag,Stefanie Andres,Einhard Kleist,Thomas F. Mentel,Franz 
Rohrer,Monika Springer,Ralf Tillmann,Jürgen Wildt,Cheng Wu,Defeng Zhao,Andreas Wahner,Astrid Kiendler-Scharr. Impact of $\mathrm{NO}<$ sub $>\mathrm{x}</$ sub $>$ on secondary organic aerosol (SOA) formation from $\alpha$-pinene and $\beta$-pinene photooxidation: the role of highly oxygenated organic nitrates[J]. Atmospheric Chemistry and Physics,2020,20(17).

[6]. Alpha and Omega Semiconductor Brings Out New High SOA MOSFETfor 12V Hot Swap Applications[J]. Manufacturing Close - Up,2020.

[7]. Alpha and Omega Semiconductor Launches New High SOA MOSFETfor 12V Hot Swap Applications[J]. Wireless News,2020.

[8]Harith Ahmad,Nur Fatini Azmy,Siti Nabila Aidit,Mohd Zamani Zulkifli. Stable multiwavelength semiconductor optical amplifier-based fiber laser using a 2-mode interferometer[J]. Microwave and Optical Technology Letters,2020,62(10).

[9]Sanghee Han,Myoseon Jang. Simulating the impact of gas-wall partitioning on SOA formation using the explicit gas mechanism integrated with aqueous reactions containing electrolytes $[\mathrm{J}]$. Science of the Total Environment,2020,748. 